\title{
Inflammation in central nervous system injury
}

\author{
Stuart M. Allan and Nancy J. Rothwell \\ School of Biological Sciences, University of Manchester, Oxford Road, Manchester M13 9PT, UK
}

\begin{abstract}
Inflammation is a key component of host defence responses to peripheral inflammation and injury, but it is now also recognized as a major contributor to diverse, acute and chronic central nervous system (CNS) disorders. Expression of inflammatory mediators including complement, adhesion molecules, cyclooxygenase enzymes and their products and cytokines is increased in experimental and clinical neurodegenerative disease, and intervention studies in experimental animals suggest that several of these factors contribute directly to neuronal injury. Most notably, specific cytokines, such as interleukin-1 (IL-1), have been implicated heavily in acute neurodegeneration, such as stroke and head injury.

In spite of their diverse presentation, common inflammatory mechanisms may contribute to many neurodegenerative disorders and in some (e.g. multiple sclerosis) inflammatory modulators are in clinical use. Inflammation may have beneficial as well as detrimental actions in the CNS, particularly in repair and recovery. Nevertheless, several anti-inflammatory targets have been identified as putative treatments for CNS disorders, initially in acute conditions, but which may also be appropriate to chronic neurodegenerative conditions.
\end{abstract}

Keywords: inflammation; cytokines; neurodegeneration; CNS; neuroimmune

\section{INFLAMMATION}

Although inflammation was recognized by the Egyptians (described in the Smith Papyrus ca. 1650 BC), it was the Roman, Cornelius Celsius ( $c a$. AD 25) who first defined it by the readily observable features of redness, swelling, heat and pain that commonly present in inflamed tissues on the surface of the body. Centuries later we have begun to understand the cellular responses and mechanisms underlying inflammation. It is now directly associated with adherence and invasion of leucocytes into injured or infected tissues, and is closely linked to activation of the immune system. Complex cascades of events that occur locally within injured tissues, and systemically have now been elucidated. These include specific mediators of inflammation such as kinins, prostanoids, substance $P$ and cytokines, as well as many physiological responses such as fever, behavioural changes and alterations in endocrine status, such as activation of the hypothalamic pituitary adrenal axis, which together constitute the acute phase response.

These host defence responses, including inflammation, are generally beneficial to the organism in limiting the survival and proliferation of invading pathogens, promoting tissue survival, repair and recovery, and conserving the energy of the organism. However, extensive, prolonged or unregulated inflammation is highly detrimental. Thus, pro-inflammatory pathways are highly regulated by an equally extensive array of anti-inflammatory processes.

The topic of this review is now extensive and would be impossible to discuss in detail. We have therefore focused

*Author for correspondence (nancy.rothwell@man.ac.uk). mainly on the role of cytokines, particularly on the role of the pro-inflammatory cytokine, interleukin-1 (IL-1).

\section{INFLAMMATION IN THE BRAIN}

The brain has several distinctive features, which mean that its response to inflammatory insults differs from other organs, and this has led to the concept of the brain as an 'immune privileged' organ. The confines of the cranium limit oedema (although this may lead to raised intracranial pressure and a reduced ventricular volume), while the tight junctions of the cerebral vasculature ('blood-brain barrier') limit the entry of large molecules and circulating cells (Petty \& Lo 2002). As an example, experimental studies in rodents demonstrate that an inflammatory stimulus (bacterial lipopolysaccharide (LPS)) induces a rapid and florid neutrophil invasion in the skin, but a very limited and delayed response in the brain (Perry et al. 1995; Matyszak 1998). Nevertheless, it is now clear that the brain does respond to peripheral inflammatory stimuli (through neural and humoral afferent signals), it integrates and regulates many aspects of the acute phase response and exhibits many local inflammatory responses that appear to contribute to both acute and chronic central nervous system (CNS) disease.

Many excellent recent reviews have discussed specific aspects of CNS inflammation, and of neurodegeneration in considerable depth (Perry et al. 1998; Gebicke-Haerter 2001; Nguyen et al. 2002). The purpose of this paper is not to cover the same ground, but instead to present an overview of the key features of inflammation, to highlight some mechanisms which may be common to seemingly diverse CNS disorders, and to discuss the limitations of current approaches, major gaps in our present knowledge and the implications and applications of recent discoveries 
to clinical disease. As stated above we will draw heavily on our own experience of the role and therapeutic potential of the cytokine interleukin-1 to illustrate some of these points, but there are now a number of equally valid targets within the inflammatory cascade.

\section{EVIDENCE FOR INFLAMMATION IN ACUTE CNS INJURY}

Acute neurodegenerative conditions such as cerebral ischaemia (e.g. stroke) and traumatic brain injury are characterized by rapid and (usually) severe insults to the brain that lead to substantial loss of nerve cells with associated functional deficits. The former is associated with abrupt cessation of blood supply to the brain as a result of a clot or haemorrhage, whereas the latter is a result of physical injury which itself often leads to secondary ischaemia. Several processes have been implicated in the ensuing damage, including increased glutamate release (excitotoxicity), oxidative stress and disturbances in ionic homeostasis. There are also substantial data demonstrating the active involvement of inflammatory processes in these diseases, much of which has been obtained from experimental studies in rodents using appropriate models of the clinical condition. This is evidence based mainly on observations of activation or invasion of inflammatory cells (e.g. microglia, leucocytes) and increased expression of transcription factors which coordinate inflammatory responses and molecules (e.g. cytokines, complement) in response to injury. However, this does not necessarily indicate a causal role, and it is important to investigate the functional consequences of increased inflammatory cells/molecules in the CNS.

Convincing evidence exists to suggest a prominent role for the cytokine IL-1 in acute neuronal injury. Increased expression of IL-1 is seen in the CNS in both the experimental and clinical setting after a variety of brain insults, and administration of exogenous IL-1 to animals undergoing ischaemic or excitotoxic challenges leads to a dramatic increase in the resulting cell death. However, perhaps the strongest support for the involvement of IL-1 derives from studies where the action of the endogenous protein has been inhibited. Central or systemic administration of the naturally occurring and highly selective IL-1 receptor antagonist (IL-1ra) markedly reduces the extent of cell death induced by ischaemic, traumatic or excitotoxic injury in the rodent brain. Similarly, inhibition of IL-1 actions by use of caspase-1 (which is required for release of active IL-1) inhibitors, caspase-1 deficient mice and anti-IL-1 antibodies, has also been shown to reduce the injury (see Rothwell \& Luheshi 2000; Allan \& Rothwell 2001).

Although not as comprehensive, there are many published studies to indicate that inhibition of other inflammatory mediators can ameliorate brain injury after acute insults. However, not all inflammatory molecules are detrimental; many have been seen to exert protective actions and block of their actions will actually enhance injury. It is important, therefore, to consider the overall balance of cytokine expression after injury, since a shift one way or the other in terms of protective versus detrimental will have a profound impact on outcome. This is exemplified by the balance between pro-inflammatory IL-1 and antiinflammatory IL-1ra (Arend 2002).

More recent studies have employed transgenic animals with specific modifications of selected genes to investigate the contribution of inflammation to acute neurodegeneration (table 1). Mice deficient in adhesion molecules (Soriano et al. 1996), pro-inflammatory cytokines (Boutin et al. 2001; Boutin \& Rothwell 2002; Ohtaki et al. 2003) and other inflammatory mediators (Iadecola et al. 1997, 2001) can show reductions in cell death in response to different insults when compared with their wild-type counterparts, while those deficient in anti-inflammatory cytokines have increased injury (Grilli et al. 2000; Boutin \& Rothwell 2002). However, this is not always the case and the reason for the discrepancies is not clear, although chronic deletion of a gene could produce a different response to that observed with acute change or there could be compensatory changes in other genes (Rudmann \& Durham 1999). This is particularly true for cytokines where there is functional overlap and redundancy (Quan \& Herkenham 2002).

\section{INFLAMMATION IN CHRONIC CNS DISEASE}

The contribution of inflammation, and in some cases of specific inflammatory mediators, has long been established in several CNS disorders such as infections (e.g. meningitis, measles, cerebral malaria and human immunodeficiency virus (HIV)) and multiple sclerosis (MS). Elegant experimental studies in rodents have identified a primary role for tumour necrosis factor- $\alpha(\mathrm{TNF} \alpha)$ in cerebral malaria, and complementary clinical studies imply a similar contribution in humans (Lou et al. 2001; de Souza \& Riley 2002). Progressive dementia is relatively common in HIV and cannot be ascribed to infection of neurons within the CNS. Instead, HIV envelope coat proteins stimulate and activate microglia, which subsequently release inflammatory mediators-most probably inflammatory cytokines (Garden 2002).

The aetiology of MS is not fully understood, but considerable evidence points towards an autoimmune condition leading to activation of the T-activator lymphocytes (and deactivation of suppressor $\mathrm{T}$ cells) which, together with macrophages, adhere to and invade the CNS (Prat et al. 2002). The key feature of MS is demyelination of axons leading to their subsequent degeneration, and formation of plaques (Bitsch et al. 2000). Animal models of MS, based primarily on immunization of rodents against myelin basic protein, can mimic many of the features of MS, but have some limitations in reproducing the chronic, relapsing and remitting nature of the disease (Link \& Xiao 2001). Nevertheless, these studies have used approaches similar to those described above to demonstrate that the cytokines $\mathrm{TNF} \alpha$ and interferon- $\gamma$ (IFN $\gamma$ ) mediate many aspects of the disease (Bettelli \& Nicholson 2000). Both $\mathrm{TNF} \alpha$ and IFN $\gamma$ are directly toxic to oligodendrocytes (Merrill et al. 1993; Vartanian et al. 1995) and, in common with other cytokines, can stimulate local inflammatory cytokine production (Benveniste \& Benos 1995; Liu et al. 1998). Clinical studies report increased expression of these cytokines (Olsson et al. 1990; Rieckmann et al. 1995), but as yet strategies to block $\mathrm{TNF}_{\alpha}$ or $\mathrm{IFN} \gamma$ directly have not been of therapeutic value; indeed the 
Table 1. Summary of ischaemic damage in cytokine knockout mice.

(tMCAO: temporary middle cerebral artery occlusion; pMCAO: permanent middle cerebral artery occlusion; HI: neonatal hypoxia/ischaemia; IL-1RAcP: IL-1 receptor accessory protein.)

\begin{tabular}{|c|c|c|c|}
\hline cytokine knock-out & treatment & $\begin{array}{c}\text { ischaemic injury } \\
\text { (compared to wild-type) }\end{array}$ & references \\
\hline \multirow[t]{2}{*}{ caspase- 1} & tMCAO & $\downarrow$ infarct ca. $-45 \%$ & Hara et al. (1997) \\
\hline & $\mathrm{HI}$ & $\downarrow$ infarct ca. $-40-53 \%$ & Liu et al. (1999) \\
\hline IL- $1 \alpha$ & $\mathrm{tMCAO}$ & no change & Boutin et al. (2001) \\
\hline IL-1 $\beta$ & tMCAO & no change & Boutin et al. (2001) \\
\hline \multirow[t]{2}{*}{ IL- $1 \alpha / \beta$} & tMCAO & $\downarrow$ infarct ca. $-70 \%$ & Boutin et al. (2001) \\
\hline & tMCAo & $\downarrow$ infarct $c a .-43 \%$ & Ohtaki et al. (2003) \\
\hline IL-1 ra & tMCAO & $\uparrow$ cortical infarct ( $c a$. eightfold) & Boutin et al. (2002) \\
\hline IL-1RI & tMCAO & no change & Touzani et al. (2002) \\
\hline IL-1RAcP & tMCAO & no change & Boutin \& Rothwell (2002) \\
\hline IL-4 & tMCAO & $\downarrow$ infarct $c a .-27 \%$ & Boutin \& Rothwell (2002) \\
\hline IL-6 & $\mathrm{tMCAO}$ & no change & Clark et al. (2000) \\
\hline IL-10 & pMCAO & $\uparrow$ infarct $c a .+30 \%$ & Grilli et al. (2000) \\
\hline IL-18 & $\mathrm{HI}$ & $\downarrow$ infarct $c a .-21 \%$ & Hedtjarn et al. (2002) \\
\hline TNF p55 & $\mathrm{tMCAO}$ & $\uparrow$ infarct $c a .+21 \%$ & Gary et al. (1998) \\
\hline TNF p75 & tMCAO & no change & Gary et al. (1998) \\
\hline TNF p55/p75 & tMCAO & $\uparrow$ infarct $c a .+18 \%$ & Gary et al. (1998) \\
\hline
\end{tabular}

exact role played by IFN $\gamma$ in the disease process is still not clear since it has been reported that IFN $\gamma$ treatment can actually reduce symptoms in the experimental allergic encephalomyelitis (EAE) model of the disease (Furlan et al. 2001). Current treatments for MS use immunosuppressant or anti-inflammatory approaches, and treatment with the cytokine interferon- $\beta$ (IFN $\beta$ ) (Hohlfeld $\&$ Wiendl 2001). The mechanisms of action of IFN $\beta$ are not fully known, but in vitro it suppresses many actions of IFN $\gamma$, inhibits the expression and release of IL- 1 and $\mathrm{TNF}_{\alpha}$, and induces release of IL-1ra (Coclet-Ninin et al. 1997; Jungo et al. 2001). One study reported that IFN $\beta$ is also neuroprotective in experimental stroke in rodents (Veldhuis et al. 2002), indicating potentially common mechanisms in these seemingly very different diseases.

Perhaps the greatest current interest in the role of inflammation in chronic CNS disorders is in Alzheimer's disease (AD). Extensive neuropathological studies reveal many of the hallmarks of inflammation in the brains of $\mathrm{AD}$ victims, including microglial activation, expression of cytokines and complement, and invasion of immune cells (McGeer \& McGeer 2002b). There has been debate over the value of anti-inflammatory drugs such as aspirin in the treatment of AD. Epidemiological studies suggest that patients on long-term anti-inflammatory treatments have a significantly reduced risk of $\mathrm{AD}$, yet prospective trials of such drugs in AD have yielded inconsistent outcomes (Zandi \& Breitner 2001; Pasinetti 2002).

Indirect evidence has implicated specific cytokines in the pathogenesis of AD (Wyss-Coray \& Mucke 2002). IL1 , for example, induces expression of amyloid precursor protein (APP), appears to alter cleavage of APP, and can cause phosphorylation of tau protein, which are the primary components of the tangles that are common in AD (Li et al. 2003). However, direct evidence for the contribution of these molecules is lacking, not least because of the difficulties in developing robust animal models that mimic the behavioural as well as pathological features of AD. Nevertheless, transgenic mice over-expressing APP carrying mutations identified in $\mathrm{AD}$ patients, exhibit some key features of AD (Janus \& Westaway 2001), and it should now be possible to cross these with mice lacking specific cytokines or look to more chronic interventions.

Polymorphisms in cytokines (notably IL-1 and IL-1ra) have been linked to early development of $\mathrm{AD}$, again suggesting a causal link between IL-1 and the disease (Griffin \& Mrak 2002). A major risk factor for AD is head injury (Lye \& Shores 2000; Jellinger et al. 2001), which induces local expression of cytokines and other inflammatory molecules (see above), and there are many parallels between vascular dementia and AD (Erkinjuntti 2002; Kalaria 2002), again highlighting a potential commonality in mechanisms of varied CNS disorders.

More recently, indirect evidence has implicated inflammation in Parkinson's disease (PD) and amyotrophic lateral sclerosis (ALS) and in the transmissible spongiform encephalopathies in animals (e.g. scrapie, bovine spongiform encephalopathy (BSE)) and humans (CreutzfeldtJakob disease (CJD)) (Maimone et al. 2001; Czlonkowska et al. 2002; McGeer \& McGeer 2002a; Van Everbroeck et al. 2002).

\section{MECHANISMS UNDERLYING INFLAMMATION AND CNS INJURY}

Most of the studies and reviews of inflammation and CNS injury to date have been either disease-specific (e.g. with focus on MS or AD), or mediator-specific (e.g. with focus on cytokines, complement or microglia). A key question is whether common mechanisms can be identified for diverse CNS disorders, and thus potentially targeted. Secondary to this is the question, if common mechanisms of inflammation do contribute to diverse forms of CNS disease, how do we explain the enormously varied aetiology, presentation and time-course? At two extremes, head injury is a rapid, accidental event that most commonly affects young people, while PD is characterized by damage to specific brain regions, motor disturbances and a chronic degenerative state. Similarly, stroke is an acute (but sometimes recurring) event that affects elderly 


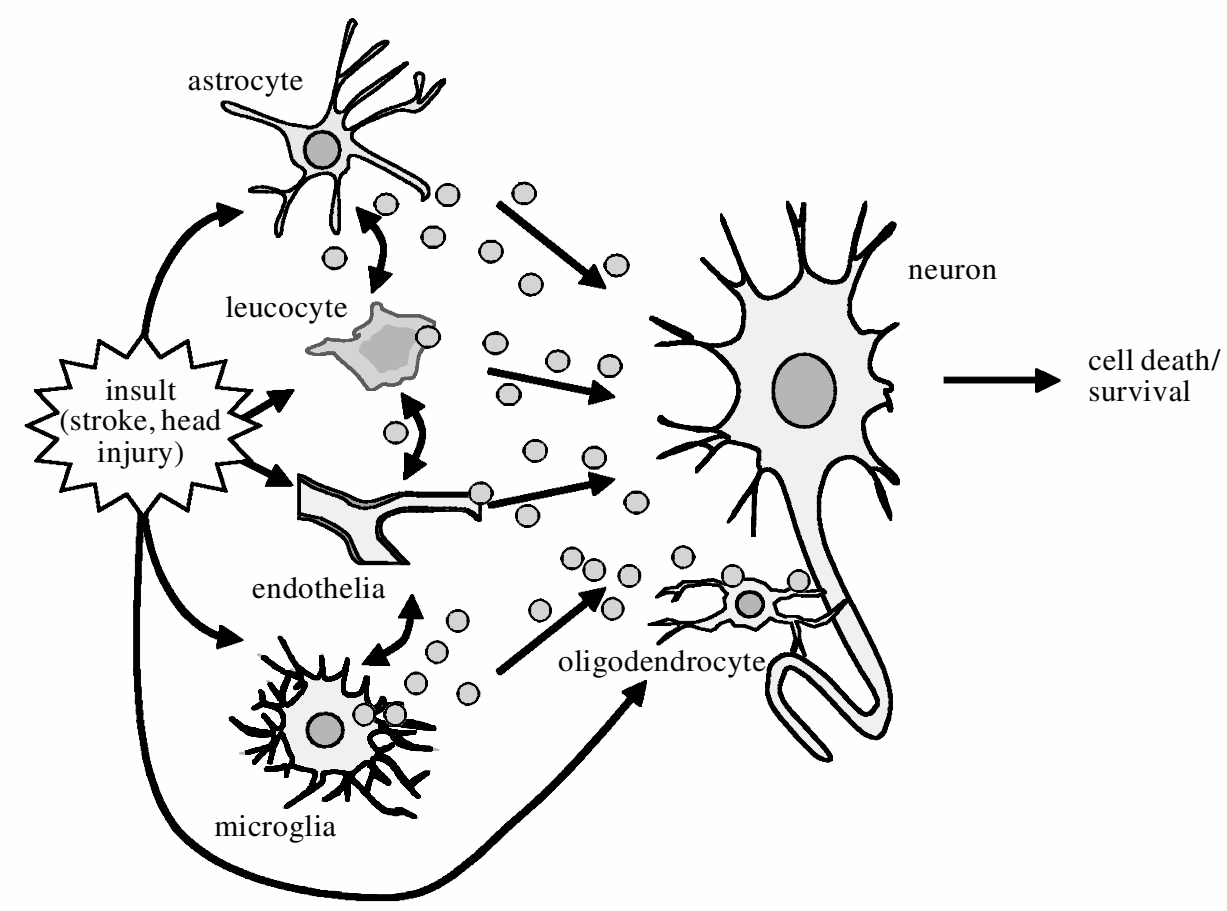

Figure 1. Overview of cells involved in cytokine release and action in neurodegeneration. Many cell types can release and respond to cytokines (grey circles) and produce direct or indirect effects on neurons that contribute to, or limit, neuronal cell death in response to different insults.

patients and causes rapid and often catastrophic events, while $\mathrm{AD}$ progresses over decades, mostly in the elderly, but occasionally presents (mainly with clearly inherited disorders) in younger people.

A broader view of these distinct diseases reveals some blurring of definitions. Competitive boxers commonly sustain repetitive head injury; many suffer traumatic brain damage, acute or delayed stroke or haemorrhage or delayed symptoms of either PD or AD (Guterman \& Smith 1987). There is a clear overlap between $\mathrm{AD}$ and vascular dementia (Erkinjuntti 2002; Kalaria 2002), and external factors such as systemic infection could have a detrimental impact on many of the CNS degenerative conditions (Perry et al. 2003).

The varied presentations of the diseases described above could be explained on the basis of the brain region primarily affected, the severity and duration of the primary insult (whether extrinsic or intrinsic) and other confounding or influencing factors. However, these putative explanations remain to be proven and the most pragmatic approach is still based on clinical diagnosis. The scientific challenge is to elucidate the common and the distinct underlying mechanisms, and thus to identify both general and disease-specific therapeutic targets.

\section{CELLS INVOLVED IN INFLAMMATION AND CNS INJURY}

Studies on CNS injury have focused predominantly on neurons. It is these cells that primarily determine CNS (and organismal) function and survival, and which (apart from perhaps rare circumstances of neurogenesis), cannot be replaced once lost. The role of other cell types or subtypes in CNS disease is becoming increasingly apparent (figure 1). White matter damage (axons) is a recognized feature of MS, but is now attracting considerable attention in stroke (Dewar et al. 1999; Goldberg \& Ransom 2003). Glia occupy the majority of the brain volume and play an active role in normal physiology and pathology (Raivich et al. 1999). The primary glial cells implicated in inflammation are microglia. These are cells of the monocyte/macrophage lineage, which are resident in the brain and are activated in response to infection, inflammation and injury (Streit 2002). Microglia are activated rapidly in response to such insults, and take on the morphology of activated macrophages (from which they are indistinguishable). They are important phagocytic cells and release numerous inflammatory molecules, particularly cytokines (Hanisch 2002). The contribution of astrocytes is more complex. These cells play key physiological roles in supporting neurons, regulating ion and transmitter concentrations and in electrical transmission, but are also an important source of neuroprotective (e.g. a number of neurotrophins) and inflammatory/potentially neurotoxic molecules (Chen \& Swanson 2003). Oligodendrocytes, the third type of glia, are critical for myelination, but are also a source of specific inflammatory molecules (Baumann \& Pham-Dinh 2001; Du \& Dreyfus 2002).

Another important impact on brain injury is the vasculature. Vascular endothelial cells are both targets and sources of inflammatory mediators (e.g. nitric oxide (NO) and prostanoids), and are particularly important in the adhesion of circulating cells of the immune system (Couraud 1994). The specific contribution of leucocytes and macrophages is now emerging. In injury settings the blood-brain barrier increases in permeability, and in some cases may break down altogether, allowing access of leucocytes into the brain parenchyma where they may release neurotoxins, activate endogenous inflammatory processes or, in the case of macrophages, phagocytose cells or cell debris (Raivich et al. 1999; del Zoppo et al. 2000). 


\section{MEDIATORS OF INFLAMMATION}

Most, if not all, of the primary mediators of inflammation identified in peripheral tissues are also expressed in the damaged CNS, and have been implicated either directly or indirectly in CNS inflammation and disease. This review does not attempt to provide a detailed analysis or discussion of each of these, but will highlight the key groups of mediators with specific focus on the cytokines (see below).

The primary mediators of inflammation such as the complement cascade, kinins, eicosanoids, $\mathrm{NO}$ and platelet activating factor (PAF), have all been reported to be expressed and/or released in response to insults to the CNS in animals and humans, and in isolated neurons or glia in vitro. Primary evidence for their involvement in CNS injury, through experimental intervention, is also available. Thus, for example, inhibition of the synthesis or action of specific components of the complement cascade, bradykinins, eicosanoids (particularly cyclooxygenase-2 enzyme (COX-2) and phospholipases), NO or PAF reduce both inflammation and neuronal injury in some experimental models (Bonventre 1997; del Zoppo et al. 2000; Gasque et al. 2000). In most cases, the contribution of these molecules to CNS injury per se (e.g. through direct influences on neuronal survival, cerebral blood flow and oxygen delivery or excitotoxicity) has not been clearly distinguished from their roles in inflammation, and clinical studies are almost exclusively descriptive. Thus evidence, if taken in isolation, is often compelling, but there are considerable gaps.

We have little information on the sequence of events or relationships between key steps in the pathways that determine the order or relative importance of putative mediators of injury. In some cases, data rely heavily on in vitro approaches or on specific interventions in vivo, and in most cases clinical data are limited or absent. Perhaps the greatest contribution has been found in the role and mechanisms of action of cytokines in CNS inflammation and injury, though even here there are many unanswered questions.

\section{CYTOKINE ACTIONS}

Cytokines have a multitude of actions in the CNS that could be important in neurodegenerative disease. These can be broken down into pro- and anti-inflammatory effects, with the latter generally viewed as neuroprotective whereas the former are mostly neurotoxic. However, it is not as simple as this, as there is significant overlap in functions between different cytokines and hence considerable redundancy. Furthermore, the role of a particular cytokine could change over time with early expression after injury contributing to the ongoing pathology, whereas later expression assists in the repair and recovery process, or vice versa. This is important to clarify, as it may be critically important in the design of therapeutic strategies to target a particular cytokine.

The effects of cytokines can depend on which cell type they act upon and whether it is a direct or indirect effect (Allan \& Rothwell 2001). Sites of action are determined by the presence of the appropriate receptor on the membrane of a particular cell. Most cytokine receptors have been described in the CNS (Szelenyi 2001), albeit for many at very low levels, although rapid upregulation can occur after injury. In terms of defining mechanisms of action, it is important to consider the experimental system used since effects observed on a single-cell population in isolation may not be mirrored in a more complex situation with an array of cell types such as is the case in vivo.

Direct actions of cytokines on neurons have mostly been reported in vitro and include changes in calcium entry, neurotransmitter release and synaptic plasticity, all of which could contribute to neuronal viability in the injured brain. Many of these effects would actually be deemed neuroprotective and this illustrates the importance of determining in vitro versus in vivo actions. It is difficult to demonstrate unequivocally the direct effect of cytokines on neurons in vivo but certain neuronal responses can certainly be modified, be it indirectly or directly, by cytokines. This includes seizure activity, which is enhanced by IL-1 administration (Vezzani et al. 1999).

In the intact brain, glia and neurons are extensively linked, and they can have profound effects on each other's function, the latter being dependent on glial support for survival. Glial cells are a primary source and target of many cytokines in the CNS, and they can release many neuroactive substances in response to cytokine stimulation, both neuroprotective and neurotoxic. These include neurotrophins and growth factors that might promote neuronal survival (Du \& Dreyfus 2002). By contrast, many potential neurotoxic substances such as NO, free radicals, proteases and excitatory amino acids can be produced by activated microglia (Raivich et al. 1999). From this it can be seen that the final outcome in terms of neuronal viability, in response to injury, will depend on both the cytokine profile and the different substances released by glial cells in response to those cytokines.

Cytokines and their receptors are present on the brain vasculature and initiation of inflammation in the brain in response to ischaemic injury occurs in the cerebral vascular endothelium (del Zoppo et al. 2000). Direct effects of cytokines on blood flow have not been reported but numerous actions have been reported on the brain vasculature that could affect neuronal viability. For example, pro-inflammatory cytokines such as IL-1 and $\mathrm{TNF} \alpha$ can cause blood-brain barrier breakdown (Quagliarello et al. 1991; Blamire et al. 2000), upregulate adhesion-molecule expression (Wong \& Dorovini-Zis 1992) and induce vasogenic oedema (Holmin \& Mathiesen 2000), as well as trigger the release of toxic substances such as NO from the vascular endothelium (Bonmann et al. 1997). The net effect of many of these actions is to increase the invasion of leucocytes into the brain parenchyma, which could contribute to injury.

\section{INFLAMMATION: BENEFICIAL OR DETRIMENTAL?}

A key question to consider in respect of the role of inflammation in CNS injury is whether it is, primarily, a good or a bad thing. In reality however, it is not as straightforward as this, since different inflammatory mediators have been shown to be detrimental, beneficial or both. Indeed many cytokines with clear detrimental effects on acute injury show beneficial actions when 
administered prior to the event in a manner similar to minor insults such as 'preconditioning' ischaemia (Tasaki et al. 1996; Jander et al. 2001). Overall, it is probably the balance between these effects that is important in determining the outcome (Nguyen et al. 2002).

In general, inflammation in the periphery, when appropriately activated and de-activated, is beneficial in the host defence response, in combating invading pathologies, isolating and removing damaged cells and promoting repair and recovery. The same may be true in CNS where limited (in severity and duration) inflammatory responses may have benefit. It is difficult to assess this benefit in evolutionary terms since most neurodegenerative disorders occur well after reproductive age and CNS injury is unlikely to have been sustainable - so such conditions are features of 'modern life'. It is possible that the inflammatory processes that benefit peripheral disease are also present in the brain, but have largely detrimental actions, particularly in the acute phase of the disease. At present, the role of inflammation and inflammatory mediators in CNS repair and recovery is unknown, but several experimental studies imply beneficial effects of specific inflammatory mediators in repair, regulation and recovery.

\section{THERAPEUTIC TARGETS AND CLINICAL RELEVANCE}

The identification of therapeutic targets depends on clear delineation of the beneficial and detrimental effects of inflammation and the roles of specific inflammatory mediators in both acute and chronic neurodegenerative states.

In acute CNS injury (e.g. stroke, brain injury), extensive data are available on specific mediators and their roles in experimental studies, largely in rodents. Here, general anti-inflammatory treatments may be beneficial in the prevention of injury. For example, both statins and aspirin have reported benefits and it is assumed that these effects are largely due to effects on cholesterol and coagulation, respectively. However, some benefit may accrue from their anti-inflammatory actions (Emsley \& Tyrrell 2002).

The most extensive evidence to date focuses on the role of IL-1 in acute neurodegeneration and the most advanced strategy is the use of IL-1ra. Clinical evidence supporting the role of IL-1 is largely indirect, including increased expression of IL-1 in CNS and of other inflammatory markers, which are induced by IL-1 in circulation. Furthermore, fever is a common and detrimental early consequence of brain damage (Ginsberg \& Busto 1998; Rossi et al. 2001), and IL-1 is one of the primary endogenous pyrogens (Dinarello 1999).

IL-1 ra is in early safety/feasibility trials on stroke and has the advantage that no adverse effects have been reported to date in animals or humans. Indeed IL-1ra is licensed for use in the treatment of rheumatoid arthritis (Bresnihan 2001). However, IL-1ra is a large molecule $(17 \mathrm{kDa})$, and while it does penetrate the brain in rodents (Gutierrez et al. 1994), a major question exists over whether brain entry is sufficient or rapid enough to offer benefit in the injured brain. Other strategies to modify IL1 bioactivity include inhibition of expression, cleavage (e.g. through caspase-1 inhibitors), soluble receptors or binding proteins (which again raise questions over brain penetration), small molecule receptor antagonists and inhibitors of signalling.

Targeting other cytokines is less well advanced, particularly since some (e.g. TNF $\alpha$, interleukin-6) have reported beneficial as well as detrimental effects (Allan \& Rothwell 2001). Molecules known as anti-inflammatory cytokines (e.g. interleukin-10, transforming growth factor$\beta$ and IFN $\beta$ ) have reported neuroprotective effects, but the experimental data are limited and side effects may prove a problem. Several other anti-inflammatory strategies are under consideration, including general inhibitors of microglial activation, chemokines, complement, NO, COX-2, neutrophil invasion and adhesion molecules. Some have entered clinical trial, but with negative or adverse outcomes (Enlimomab Acute Stroke Trial Investigators 2001; Legos et al. 2002; Narayan et al. 2002).

The failure of numerous clinical trials in stroke and head injury is now an issue for major concern. In some cases, the reasons are clear and have included insufficient preclinical data, limited efficacy of the test compound, unacceptable side effects or poorly designed clinical trials (Stroke Therapy Academic Industry Roundtable 1999; Narayan et al. 2002; Fisher \& Brott 2003). Nevertheless, these many failures do question the relevance of currently available experimental studies to clinical brain injury and the general strategy for clinical trial design. It may prove necessary to undertake more extensive preclinical research in higher species including primates, which raises obvious ethical issues. It may also be necessary to conduct more focused and intensive clinical studies on newly defined patient groups to test 'proof of principle' before considering the general ability of any treatment for wider patient participation.

Identification of valuable therapeutic anti-inflammatory targets for chronic CNS disorders is somewhat limited by the experimental models available. IFN $\beta$, in current use for MS, is probably dependent on inhibition of inflammation, and COX-2 inhibitors may become generally used in AD. However, the chronic benefits of IFN $\beta$ are limited or absent. For other chronic disorders, further evidence is required, but anti-inflammatory approaches, if safe and if shown to have efficacy in acute disease, may be tested in chronic disorders.

\section{CONCLUSIONS/FUTURE RESEARCH}

Inflammation in the brain and its contribution to CNS injury is a relatively new area of research and clinical interest, but is now the focus of extensive investigation. Inflammation, though beneficial in many diseases, is not a feature of healthy tissue. Thus, specific anti-inflammatory treatments are unlikely to have adverse effects on normal function. This is in contrast to the many therapeutic approaches tested or used to date such as glutamate or calcium antagonists, inhibitors of sodium channels, or modifiers of dopaminergic, serotonergic or cholinergic function. All of these processes are essential for normal function, so modification may have unwanted effects. Nevertheless there remain many unanswered questions about the relative importance of specific mediators of inflammation, their beneficial as well as detrimental effects, and the possibility of redundancy, since many cytokines have similar actions. We know relatively little 
about the mechanisms of action of inflammation in CNS disease and experimental studies have often focused on in vitro approaches or on very short outcomes after interventions on in vivo experimental models.

A rapidly emerging area is the study of polymorphisms in cytokine genes, particularly IL-1 and $\mathrm{TNF} \alpha$, which have been linked to CNS disorders (Kanemoto et al. 2000; McGeer \& McGeer 2001; McGeer et al. 2002; Schulte et al. 2002). These studies are likely to yield valuable information on the mediators of CNS disorders and possible identification of patients 'at risk' who may therefore benefit from early treatment. By contrast, we know little about the impact of environmental factors on susceptibility to inflammation or the interactions between environment and genes.

Finally, we need to consider which patients should be targeted and the likely benefits. For example, a reduction of mortality in stroke or head injury, which results in more severely disabled patients or prolongation of the endstages of chronic CNS disorders, is of questionable benefit. These may be physiological and sociological rather than scientific issues, but ones that should be on the agenda for all scientists.

\section{REFERENCES}

Allan, S. M. \& Rothwell, N. J. 2001 Cytokines and acute neurodegeneration. Nat. Rev. Neurosci. 2, 734-744.

Arend, W. P. 2002 The balance between IL-1 and IL-1Ra in disease. Cytokine Growth Factor Rev. 13, 323-340.

Baumann, N. \& Pham-Dinh, D. 2001 Biology of oligodendrocyte and myelin in the mammalian central nervous system. Physiol. Rev. 81, 871-927.

Benveniste, E. N. \& Benos, D. J. 1995 TNF- $\alpha-$ and IFN- $\gamma-$ mediated signal transduction pathways: effect on glial cell gene expression and function. FASEB f. 9, 1577-1584.

Bettelli, E. \& Nicholson, L. B. 2000 The role of cytokines in experimental autoimmune encephalomyelitis. Arch. Immunol. Ther. Exp. (Warsz.) 48, 389-398.

Bitsch, A., Schuchardt, J., Bunkowski, S., Kuhlmann, T. \& Bruck, W. 2000 Acute axonal injury in multiple sclerosis. Correlation with demyelination and inflammation. Brain 123, 1174-1183.

Blamire, A. M., Anthony, D. C., Rajagopalan, B., Sibson, N. R., Perry, V. H. \& Styles, P. 2000 Interleukin-1betainduced changes in blood-brain barrier permeability, apparent diffusion coefficient, and cerebral blood volume in the rat brain: a magnetic resonance study. F. Neurosci. 20, 8153-8159.

Bonmann, E., Suschek, C., Spranger, M. \& Kolb-Bachofen, V. 1997 The dominant role of exogenous or endogenous interleukin-1 beta on expression and activity of inducible nitric oxide synthase in rat microvascular brain endothelial cells. Neurosci. Lett. 230, 109-112.

Bonventre, J. V. 1997 Roles of phospholipases A2 in brain cell and tissue injury associated with ischemia and excitotoxicity. f. Lipid Med. Cell Signal. 16, 199-208.

Boutin, H. \& Rothwell, N. J. 2002 Cerebral ischaemic processes and cytokines: can transgenic mice help? In Pharmacology of cerebral ischaemia (ed. J. Krieglstein \& S. Klumpp), pp. 183-190. Stuttgart: Medpharm.

Boutin, H., LeFeuvre, R. A., Horai, R., Asano, M., Iwakura, Y. \& Rothwell, N. J. 2001 Role of IL-1alpha and IL-1beta in ischemic brain damage. F. Neurosci. 21, 5528-5534.

Bresnihan, B. 2001 The safety and efficacy of interleukin-1 receptor antagonist in the treatment of rheumatoid arthritis. Sem. Arthritis Rheum. 30, 17-20.
Chen, Y. \& Swanson, R. A. 2003 Astrocytes and brain injury. 7. Cereb. Blood Flow Metab. 23, 137-149.

Clark, W. M., Rinker, L. G., Lessov, N. S., Hazel, K., Hill, J. K., Stenzel-Poore, M. \& Eckenstein, F. 2000 Lack of interleukin-6 expression is not protective against focal central nervous system ischemia. Stroke 31, 1715-1720.

Coclet-Ninin, J., Dayer, J. M. \& Burger, D. 1997 Interferonbeta not only inhibits interleukin-1beta and tumor necrosis factor-alpha but stimulates interleukin-1 receptor antagonist production in human peripheral blood mononuclear cells. Eur. Cytokine Netw. 8, 345-349.

Couraud, P.-O. 1994 Interactions between lymphocytes, macrophages, and central nervous system cells. F. Leukocyte Biol. 56, 407-415.

Czlonkowska, A., Kurkowska-Jastrzebska, I., Czlonkowski, A., Peter, D. \& Stefano, G. B. 2002 Immune processes in the pathogenesis of Parkinson's disease-a potential role for microglia and nitric oxide. Med. Sci. Monit. 8, RA165RA177.

del Zoppo, G., Ginis, I., Hallenbeck, J. M., Iadecola, C., Wang, X. \& Feuerstein, G. Z. 2000 Inflammation and stroke: putative role for cytokines, adhesion molecules and iNOS in brain response to ischeamia. Brain Pathol. 10, 95-112.

de Souza, J. B. \& Riley, E. M. 2002 Cerebral malaria: the contribution of studies in animal models to our understanding of immunopathogenesis. Microbes Infect. 4, 291-300.

Dewar, D., Yam, P. \& McCulloch, J. 1999 Drug development for stroke: importance of protecting cerebral white matter. Eur. F. Pharmacol. 375, 41-50.

Dinarello, C. A. 1999 Cytokines as endogenous pyrogens. F. Infect. Dis. 179, s294-s304.

Du, Y. \& Dreyfus, C. F. 2002 Oligodendrocytes as providers of growth factors. F. Neurosci. Res. 68, 647-654.

Emsley, H. C. \& Tyrrell, P. J. 2002 Inflammation and infection in clinical stroke. F. Cereb. Blood Flow Metab. 22, 1399-1419.

Enlimomab Acute Stroke Trial Investigators 2001 Use of antiICAM-1 therapy in ischemic stroke: results of the Enlimomab Acute Stroke Trial. Neurology 57, 1428-1434.

Erkinjuntti, T. 2002 Diagnosis and management of vascular cognitive impairment and dementia. F. Neural Transm. Suppl. 63, 91-109.

Fisher, M. \& Brott, T. G. 2003 Emerging therapies for acute ischemic stroke: new therapies on trial. Stroke 34, 359-361.

Furlan, R. (and 10 others) 2001 Intrathecal delivery of IFNgamma protects $\mathrm{C} 57 \mathrm{BL} / 6$ mice from chronic-progressive experimental autoimmune encephalomyelitis by increasing apoptosis of central nervous system-infiltrating lymphocytes. f. Immunol. 167, 1821-1829.

Garden, G. A. 2002 Microglia in human immunodeficiency virus-associated neurodegeneration. Glia 40, 240-251.

Gary, D. S., Bruce-Keller, A. J., Kindy, M.S. \& Mattson, M. P. 1998 Ischemic and excitotoxic brain injury is enhanced in mice lacking the p55 tumor necrosis factor receptor. F. Cereb. Blood Flow Metab. 18, 1283-1287.

Gasque, P., Dean, Y. D., McGreal, E. P., VanBeek, J. \& Morgan, B. P. 2000 Complement components of the innate immune system in health and disease in the CNS. Immunopharmacology 49, 171-186.

Gebicke-Haerter, P. J. 2001 Microglia in neurodegeneration: molecular aspects. Microsc. Res. Tech. 54, 47-58.

Ginsberg, M. D. \& Busto, R. 1998 Combating hyperthermia in acute stroke: a significant clinical concern. Stroke 29, 529-534.

Goldberg, M. P. \& Ransom, B. R. 2003 New light on white matter. Stroke 34, 330-332.

Griffin, W. S. \& Mrak, R. E. 2002 Interleukin-1 in the genesis and progression of and risk for development of neuronal 
degeneration in Alzheimer's disease. F. Leukoc. Biol. 72, 233-238.

Grilli, M., Barbieri, I., Basudev, H., Brusa, R., Casati, C., Lozza, G. \& Ongini, E. 2000 Interleukin-10 modulates neuronal threshold of vulnerability to ischaemic damage. Eur. f. Neurosci. 12, 2265-2272.

Guterman, A. \& Smith, R. W. 1987 Neurological sequelae of boxing. Sports Med. 4, 194-210.

Gutierrez, E. G., Banks, W. A. \& Kastin, A. J. 1994 Bloodborne interleukin-1 receptor antagonist crosses the bloodbrain barrier. F. Neuroimmunol. 55, 153-160.

Hanisch, U. K. 2002 Microglia as a source and target of cytokines. Glia 40, 140-155.

Hara, H., Fink, K., Endres, M., Friedlander, R. M., Gagliardini, V., Yuan, J. Y. \& Moskowitz, M. A. 1997 Attenuation of transient focal cerebral ischemic injury in transgenic mice expressing a mutant ICE inhibitory protein. f. Cereb. Blood Flow Metab. 17, 370-375.

Hedtjarn, M., Leverin, A. L., Eriksson, K., Blomgren, K., Mallard, C. \& Hagberg, H. 2002 Interleukin-1 8 involvement in hypoxic-ischemic brain injury. F. Neurosci. 22, 59105919.

Hohlfeld, R. \& Wiendl, H. 2001 The ups and downs of multiple sclerosis therapeutics. Ann. Neurol. 49, 281-284.

Holmin, S. \& Mathiesen, T. 2000 Intracerebral administration of interleukin-1beta and induction of inflammation, apoptosis, and vasogenic edema. F. Neurosurg. 92, 108-120.

Iadecola, C., Zhang, F., Casey, R., Nagayama, M. \& Ross, M. E. 1997 Delayed reduction of ischemic brain injury and neurological deficits in mice lacking the inducible nitric oxide synthase gene. F. Neurosci. 17, 9157-9164.

Iadecola, C., Niwa, K., Nogawa, S., Zhao, X., Nagayama, M., Araki, E., Morham, S. \& Ross, M. E. 2001 Reduced susceptibility to ischemic brain injury and $N$-methyl-D-aspartatemediated neurotoxicity in cyclooxygenase-2-deficient mice. Proc. Natl Acad. Sci. USA 98, 1294-1299.

Jander, S., Schroeter, M., Peters, O., Witte, O. W. \& Stoll, G. 2001 Cortical spreading depression induces proinflammatory cytokine gene expression in the rat brain. f. Cereb. Blood Flow Metab. 21, 218-225.

Janus, C. \& Westaway, D. 2001 Transgenic mouse models of Alzheimer's disease. Physiol. Behav. 73, 873-886.

Jellinger, K. A., Paulus, W., Wrocklage, C. \& Litvan, I. 2001 Effects of closed traumatic brain injury and genetic factors on the development of Alzheimer's disease. Eur. F. Neurol. 8, 707-710.

Jungo, F., Dayer, J. M., Modoux, C., Hyka, N. \& Burger, D. 2001 IFN-beta inhibits the ability of $\mathrm{T}$ lymphocytes to induce TNF-alpha and IL-1beta production in monocytes upon direct cell-cell contact. Cytokine 14, 272-282.

Kalaria, R. 2002 Similarities between Alzheimer's disease and vascular dementia. F. Neurol. Sci. 203-204, 29-34.

Kanemoto, K., Kawasaki, J., Miyamoto, T., Obayashi, H. \& Nishimura, M. 2000 Interleukin (IL) 1beta, IL-1alpha and IL-1 receptor antagonist gene polymorphisms in patients with temporal lobe epilepsy. Ann. Neurol. 47, 571-574.

Legos, J. J., Tuma, R. F. \& Barone, F. C. 2002 Pharmacological interventions for stroke: failures and future. Expert Opin. Invest. Drugs 11, 603-614.

Li, Y., Liu, L., Barger, S. W. \& Griffin, W. S. 2003 Interleukin-1 mediates pathological effects of microglia on tau phosphorylation and on synaptophysin synthesis in cortical neurons through a p38-MAPK pathway. F. Neurosci. 23, 1605-1611.

Link, H. \& Xiao, B. G. 2001 Rat models as tool to develop new immunotherapies. Immunol. Rev. 184, 117-128.

Liu, J. S. H., Amaral, T. D., Brosnan, C. F. \& Lee, S. C. 1998 IFNs are critical regulators of IL-1 receptor antagonist and
IL-1 expression in human microglia. f. Immunol. 161, 1989-1996.

Liu, X. H., Kwon, D., Schielke, G. P., Yang, G. Y., Silverstein, F. S. \& Barks, J. D. 1999 Mice deficient in interleukin-1 converting enzyme are resistant to neonatal hypoxicischemic brain damage. f. Cereb. Blood Flow Metab. 19, 1099-1108.

Lou, J., Lucas, R. \& Grau, G. E. 2001 Pathogenesis of cerebral malaria: recent experimental data and possible applications for humans. Clin. Microbiol. Rev. 14, 810-820.

Lye, T. C. \& Shores, E. A. 2000 Traumatic brain injury as a risk factor for Alzheimer's disease: a review. Neuropsychol. Rev. 10, 115-129.

McGeer, P. L. \& McGeer, E. G. 2001 Polymorphisms in inflammatory genes and the risk of Alzheimer disease. Arch. Neurol. 58, 1790-1792.

McGeer, P. L. \& McGeer, E. G. $2002 a$ Inflammatory processes in amyotrophic lateral sclerosis. Muscle Nerve 26, 459-470.

McGeer, P. L. \& McGeer, E. G. $2002 b$ Local neuroinflammation and the progression of Alzheimer's disease. F. Neurovirol. 8, 529-538.

McGeer, P. L., Yasojima, K. \& McGeer, E. G. 2002 Association of interleukin-1 beta polymorphisms with idiopathic Parkinson's disease. Neurosci. Lett. 326, 67-69.

Maimone, D., Dominici, R. \& Grimaldi, L. M. 2001 Pharmacogenomics of neurodegenerative diseases. Eur. F. Pharmacol. 413, 11-29.

Matyszak, M. K. 1998 Inflammation in the CNS: balance between immunological privilege and immune responses. Prog. Neurobiol. 56, 19-35.

Merrill, J. E., Ignarro, L. J., Sherman, M. P., Melinek, J. \& Lane, T. E. 1993 Microglial cell cytotoxicity of oligodendrocytes is mediated through nitric oxide. f. Immunol. 151, 2132-2141.

Narayan, R. K. (and 45 others) 2002 Clinical trials in head injury. F. Neurotrauma 19, 503-557.

Nguyen, M. D., Julien, J. P. \& Rivest, S. 2002 Innate immunity: the missing link in neuroprotection and neurodegeneration? Nat. Rev. Neurosci. 3, 216-227.

Ohtaki, H. (and 10 others) 2003 Suppression of oxidative neuronal damage after transient middle cerebral artery occlusion in mice lacking interlekin-1. Neurosci. Res. 45, 313-324.

Olsson, T., Zhi, W. W., Hojeberg, B., Kostulas, V., Jiang, Y. P., Anderson, G., Ekre, H. P. \& Link, H. 1990 Autoreactive $\mathrm{T}$ lymphocytes in multiple sclerosis determined by antigen-induced secretion of interferon-gamma. f. Clin. Invest. 86, 981-985.

Pasinetti, G. M. 2002 From epidemiology to therapeutic trials with anti-inflammatory drugs in Alzheimer's disease: the role of NSAIDs and cyclooxygenase in beta-amyloidosis and clinical dementia. F. Alzheimer's Dis. 4, 435-445.

Perry, V. H., Bell, M. D., Brown, H. C. \& Matyszak, M. K. 1995 Inflammation in the nervous system. Curr. Opin. Neurobiol. 5, 636-641.

Perry, V.H., Bolton, S. J., Anthony, D.C. \& Betmouni, S. 1998 The contribution of inflammation to acute and chronic neurodegeneration. Res. Immunol. 149, 721-725.

Perry, V. H., Newman, T. A. \& Cunningham, C. 2003 The impact of systemic infection on the progression of neurodegenerative disease. Nat. Rev. Neurosci. 4, 103-112.

Petty, M. A. \& Lo, E. H. 2002 Junctional complexes of the blood-brain barrier: permeability changes in neuroinflammation. Prog. Neurobiol. 68, 311-323.

Prat, A., Biernacki, K., Lavoie, J. F., Poirier, J., Duquette, P. \& Antel, J. P. 2002 Migration of multiple sclerosis lymphocytes through brain endothelium. Arch. Neurol. 59, 391-397.

Quagliarello, V. J., Wispelwey, B., Long Jr, W. J. \& Scheld, W. M. 1991 Recombinant human interleukin-1 induces 
meningitis and blood-brain barrier injury in the rat. Characterization and comparison with tumor necrosis factor. $\mathcal{F}$. Clin. Invest. 87, 1360-1366.

Quan, N. \& Herkenham, M. 2002 Connecting cytokines and brain: a review of current issues. Histol. Histopathol. 17, 273-288.

Raivich, G., Bohatschek, M., Kloss, C. U., Werner, A., Jones, L. L. \& Kreutzberg, G. W. 1999 Neuroglial activation repertoire in the injured brain: graded response, molecular mechanisms and cues to physiological function. Brain Res. Brain Res. Rev. 30, 77-105.

Rieckmann, P., Albrecht, M., Kitze, B., Weber, T., Tumani, H., Broocks, A., Luer, W., Helwig, A. \& Poser, S. 1995 Tumor necrosis factor-alpha messenger RNA expression in patients with relapsing-remitting multiple sclerosis is associated with disease activity. Ann. Neurol. 37, 82-88.

Rossi, S., Zanier, E. R., Mauri, I., Columbo, A. \& Stocchetti, N. 2001 Brain temperature, body core temperature, and intracranial pressure in acute cerebral damage. f. Neurol. Neurosurg. Psychiat. 71, 448-454.

Rothwell, N. J. \& Luheshi, G. N. 2000 Interleukin 1 in the brain: biology, pathology and therapeutic target. Trends Neurosci. 23, 618-625.

Rudmann, D. G. \& Durham, S. K. 1999 Utilization of genetically altered animals in the pharmaceutical industry. Toxicol. Pathol. 27, 111-114.

Schulte, T., Schols, L., Muller, T., Woitalla, D., Berger, K. \& Kruger, R. 2002 Polymorphisms in the interleukin-1 alpha and beta genes and the risk for Parkinson's disease. Neurosci. Lett. 326, 70-72.

Soriano, S. G., Lipton, S. A., Wang, Y. F., Xiao, M., Springer, T. A., Gutierrez-Ramos, J.-C. \& Hickey, P. R. 1996 Intercellular adhesion molecule-1-deficient mice are less susceptible to cerebral ischemia-reperfusion injury. Ann. Neurol. 39, 618-624.

Streit, W. J. 2002 Microglia as neuroprotective, immunocompetent cells of the CNS. Glia 40, 133-139.

Stroke Therapy Academic Industry Roundtable 1999 Recommendations for standards regarding preclinical neuroprotec- tive and restorative drug development. Stroke 30, 2752 2758.

Szelenyi, J. 2001 Cytokines and the central nervous system. Brain Res. Bull. 54, 329-338.

Tasaki, K., Nawashiro, H., Ohtsuki, T., Ruetzler, C., Martin, D. \& Hallenbeck, J. M. 1996 Cytokines in the induction of tolerance to ischemia. In Pharmacology of cerebral ischemia (ed. J. Krieglstein), pp. 1-8. Stuttgart: Medpharm.

Touzani, O., Boutin, H., LeFeuvre, R., Parker, L., Miller, A., Luheshi, G. \& Rothwell, N. 2002 Interleukin-1 influences ischemic brain damage in the mouse independently of the interleukin-1 type I receptor. F. Neurosci. 22, 38-43.

Van Everbroeck, B., Dewulf, E., Pals, P., Lubke, U., Martin, J. J. \& Cras, P. 2002 The role of cytokines, astrocytes, microglia and apoptosis in Creutzfeldt-Jakob disease. Neurobiol. Aging 23, 59-64.

Vartanian, T., Li, Y., Zhao, M. \& Stefansson, K. 1995 Interferon- $\gamma$-induced oligodendrocyte cell death: implications for the pathogenesis of multiple sclerosis. Mol. Med. 1, 732-743.

Veldhuis, W. B., van der Melde, P. H., Bär, D. \& Nicolay, K. 2002 Delayed treatment with interferon- $\beta$ protects against ischemic stroke. Stroke 33, 346.

Vezzani, A., Conti, N., De Luigi, A., Ravizza, T., Moneta, D., Marchesi, F. \& De Simoni, M. G. 1999 Interleukin-1 $\beta$ immunoreactivity and microglia are enhanced in the rat hippocampus by focal kainate application: functional evidence for enhancement of electrographic seizures. F. Neurosci. 19, 5054-5065.

Wong, D. \& Dorovini-Zis, K. 1992 Upregulation of intercellular adhesion molecule-1 (ICAM-1) expression in primary cultures of human brain microvessel endothelial cells by cytokines and lipopolysaccharide. F. Neuroimmunol. 39, 11-21.

Wyss-Coray, T. \& Mucke, L. 2002 Inflammation in neurodegenerative disease-a double-edged sword. Neuron 35, 419432.

Zandi, P. P. \& Breitner, J. C. 2001 Do NSAIDs prevent Alzheimer's disease? And, if so, why? The epidemiological evidence. Neurobiol. Aging 22, 811-817. 